\title{
TUBERCULOSIS
}

\section{Paradoxical reactions during tuberculosis treatment in patients with and without HIV co-infection}

\author{
R A M Breen, C J Smith, H Bettinson, S Dart, B Bannister, M A Johnson, M C I Lipman
}

Thorax 2004;59:704-707. doi: 10.1136/thx.2003.019224

See end of article for authors' affiliations

.....................

Correspondence to:

Dr R Breen, Department of

Thoracic and HIV

Medicine, Royal Free

Hospital, London NW3

2QG, UK; rambreen@

doctors.org.uk

Received

21 November 2003

Accepted 22 April 2004
Background: It has been suggested that deterioration of tuberculosis (TB) during appropriate treatment, termed a paradoxical reaction (PR), is more common and severe in HIV positive individuals on highly active antiretroviral therapy (HAART).

Method: A study was undertaken to determine the frequency of PR and its associated features in a population of $\mathrm{HIV}+\mathrm{TB}+$ patients and a similar sized group of $\mathrm{HIV}-\mathrm{TB}+$ individuals.

Results: PR occurred in $28 \%$ of $50 \mathrm{HIV}+\mathrm{TB}+$ patients and $10 \%$ of $50 \mathrm{HIV}-\mathrm{TB}+$ patients. Disseminated TB was present in eight of $13 \mathrm{HIV}+\mathrm{TB}+$ patients and four of five $\mathrm{HIV}-\mathrm{TB}+$ patients with PR. In $28 \mathrm{HIV}+\mathrm{TB}+$ patients starting HAART, PR was significantly associated with commencing HAART within 6 weeks of starting antituberculosis treatment $(p=0.03)$ and was more common in those with disseminated TB $(p=0.09)$. No association was found between development of PR and baseline CD4 count or CD4 response to HAART.

Conclusions: PR is common in HIV infected and uninfected individuals with TB. Early introduction of HAART and the presence of disseminated TB appear to be important in co-infected patients.
$\mathrm{T}$ he phenomenon of a "paradoxical reaction" (PR) during the treatment of tuberculosis (TB), in which existing disease may worsen or new lesions appear, has been recognised for many years. ${ }^{12}$ It is unpredictable in its timing (occurring anything from a few days to many months after the start of antituberculosis chemotherapy) and in its duration and severity. Recognition of deterioration resulting from PR rather than from treatment failure, drug resistance or another infection can therefore be difficult. Most reported cases have complicated the treatment of lymph node or cerebral disease, with enlargement of nodes seen in approximately $30 \%$ in one large series. ${ }^{3}$ While these occurrences are usually self-limiting, respiratory failure and death have been described, mainly with miliary and pulmonary disease. ${ }^{4}$ In the past few years an increase in the frequency and severity of PR in association with HIV co-infection has been reported compared with both HIV negative TB patients and co-infected patients before the introduction of antiretroviral drugs. ${ }^{5}$ The reasons for this are not clear but may be related to the introduction of highly active antiretroviral therapy (HAART) which has been implicated in inflammatory reactions analogous to PR in patients with Pneumocystis pneumonia, ${ }^{6}$ Mycobacterium avium complex, ${ }^{7}$ and cytomegalovirus infection. ${ }^{8}$ However, not all studies performed since the introduction of HAART have reported an increased frequency in PR. ${ }^{9}$ This may be partly because investigators report predominantly either outpatients or inpatients. This could lead to differences between cohorts in, for example, TB severity and drug adherence-which may in turn alter the occurrence of PR. The drug regimens in studies published so far have also largely avoided the use of protease inhibitors or non-nucleoside inhibitors with rifamycins, which would now be considered standard treatment.

To estimate the frequency and nature of $\mathrm{PR}$ in a heterogeneous clinic and inpatient population we performed a retrospective study of all HIV/TB co-infected individuals treated at our institution from 1997 when HAART became widely available. A similar sized group of HIV negative TB patients served as a comparison population.

\section{METHODS}

Patients diagnosed with TB between February 1997 and February 2002 were identified from the Royal Free Hospital TB database which records all patients diagnosed with TB in our urban teaching hospital. Treatment was initiated either as an inpatient or an outpatient depending on symptom severity. All patients in the clinic received self-administered treatment. Clinical information was validated against patient case notes. Diagnoses were accepted if the patient had: (1) a positive culture for Mycobacterium tuberculosis; (2) had been culture negative but AFB smear and/or nucleic acid amplification assay positive (TB Strand Displacement Amplification assay, Becton-Dickinson, NJ, USA) with clinicoradiological features and response to treatment consistent with TB; (3) histological findings consistent with TB and response to treatment consistent with TB. Patients were excluded if they were followed up for less than 6 months or never started TB treatment. The comparator population was selected by identifying consecutive TB patients who satisfied the definition of diagnosis, did not meet the exclusion criteria, and had a documented negative HIV antibody test.

PR was defined as a worsening of clinical or radiological findings following the initiation of appropriate antituberculous treatment in the absence of evidence of disease relapse or the presence of another diagnosis. Dissemination was defined as clinically or radiologically apparent disease at more than one site, with or without laboratory confirmation. Dual antiretroviral therapy was defined as the use of two nucleoside reverse-transcriptase inhibitors (NRTIs). HAART involved the use of two NRTIs with either a protease inhibitor (PI) or a non-nucleoside reverse-transcriptase inhibitor (NNRTI).

Statistical analysis was performed using Fisher's exact test, $\chi^{2}$ test, Mann-Whitney U test, and Kaplan-Meier methods.

\section{RESULTS}

Sixty four patients with HIV/TB co-infection were identified, 14 of whom were excluded from the study: four were treated on clinical grounds but did not meet our diagnostic criteria 


\begin{tabular}{|c|c|c|c|}
\hline & $\begin{array}{l}\text { HIV +ve } \\
(n=50)\end{array}$ & $\begin{array}{l}\text { HIV - ve } \\
(n=50)\end{array}$ & p value* \\
\hline Median (range) age (years) & $36(24-62)$ & $32(16-69)$ & 0.2 \\
\hline Sex (male) & $26(52 \%)$ & $26(52 \%)$ & 0.9 \\
\hline \multicolumn{4}{|l|}{ Primary site of disease } \\
\hline Pulmonary & $25(50 \%)$ & $19(38 \%)$ & 0.03 \\
\hline Lymph node & $15(30 \%)$ & $15(30 \%)$ & \\
\hline CNS & $1(2 \%)$ & $8(16 \%)$ & \\
\hline Pleural & $1(2 \%)$ & $4(8 \%)$ & \\
\hline Miliary & $4(8 \%)$ & $0(0 \%)$ & \\
\hline Other & $4(8 \%)$ & $4(8 \%)$ & \\
\hline Disseminated TB & $20(40 \%)$ & $6(12 \%)$ & 0.001 \\
\hline \multicolumn{4}{|l|}{ Ethnicity } \\
\hline African & $35(70 \%)$ & $21(42 \%)$ & $<0.0001$ \\
\hline Asian & $2(4 \%)$ & $19(38 \%)$ & \\
\hline Other & $13(26 \%)$ & $10(20 \%)$ & \\
\hline TB culture positive & $42(84 \%)$ & $31(62 \%)$ & 0.06 \\
\hline
\end{tabular}

(one of whom experienced PR), four had died (three within a few days of starting TB treatment and one of non-Hodgkin's lymphoma without adhering to antituberculosis treatment), in three cases patient records were unavailable for data analysis, and three patients were followed up at other centres. This left 50 HIV+TB+ individuals. Fifty consecutive HIV-TB+ patients fitting the inclusion criteria were identified for inclusion in the control group. The baseline characteristics of the two groups are shown in table 1. The two groups differed significantly with respect to both ethnicity $(p<0.001)$ and the presence of disseminated TB $(p=0.001)$.

Paradoxical reactions were seen in $14 \mathrm{HIV}+\mathrm{TB}+$ patients (28\%; $95 \%$ CI 16 to 43 ) and five HIV-TB+ patients ( $10 \%$; $95 \%$ CI 3 to 22). All cases of PR were in patients with culture confirmed TB sensitive to all first line antituberculosis drugs. PR occurred in patients of all ethnicities. The features associated with PR in the two groups are shown in table 2. In those with $\mathrm{PR}$, disseminated $\mathrm{TB}$ was present in nine of 14 $(65 \%)$ HIV+TB+ patients and four of five $(80 \%)$ HIV-TB+ patients. The median time from starting TB treatment to PR was 33 days in the HIV+TB+ patients (range 3-173 days) and 87 days in the HIV-TB+ patients (range 23-157 days). However, in HIV+TB+ patients the median time between starting HAART and PR was only 11 days. Of the HIV+TB+ individuals with PR, all were either receiving HAART at the time of TB diagnosis or started it subsequent to this diagnosis. Two patients had episodes of PR before starting HAART, one of whom had a further episode after starting antiretroviral therapy.

Corticosteroids were prescribed in nine cases. The reasons for the prescription were severe systemic manifestations or prolonged duration of PR. Treatment led to an improvement in all cases in a median time of 3 days (range 1-7). Effective doses of prednisolone ranged from 10 to $80 \mathrm{mg}$ daily. In all cases steroids were tailed off gradually, with PR recurring in three patients on their discontinuation. All responded again when steroids were restarted.

Serum levels of C-reactive protein (CRP), erythrocyte sedimentation rate, albumin, lactate dehydrogenase (LDH), and haemoglobin as markers of systemic inflammation were obtained in HIV+TB+ patients at the time of TB diagnosis. No significant differences in these parameters were observed when comparing patients who subsequently developed PR with those who did not (data not shown). At the time of PR, CRP levels were increased in all 10 in whom they were measured and LDH levels were increased in five of seven in whom they were measured. However, only in two cases had these levels risen in comparison with the levels at TB diagnosis.
Table 2 Comparison of frequency, manifestations, and treatment of paradoxical reactions (PR) between $\mathrm{HIV}+\mathrm{TB}+$ and $\mathrm{HIV}-\mathrm{TB}+$ patients

\begin{tabular}{|c|c|c|}
\hline & $\begin{array}{l}\text { HIV+ve } \\
(n=50)\end{array}$ & $\begin{array}{l}\text { HIV-ve } \\
(n=50)\end{array}$ \\
\hline PR & $14(28 \%)$ & $5(10 \%)$ \\
\hline Disseminated TB and PR & $9(65 \%)$ & $4(80 \%)$ \\
\hline $\begin{array}{l}\text { Median (range) time from starting } \\
\text { TB medication to onset of PR (days) }\end{array}$ & $33(3-173)$ & $87(23-157)$ \\
\hline $\begin{array}{l}\text { Median (range) time from starting } \\
\text { HAART to onset of PR (days) }\end{array}$ & $11(8-18)$ & NA \\
\hline $\begin{array}{l}\text { PR treated with corticosteroids } \\
\text { Manifestations of PR }\end{array}$ & 8 & 1 \\
\hline Fever & 4 & 2 \\
\hline Ascites & 1 & 0 \\
\hline Lymph nodes & 7 & 2 \\
\hline CNS & 2 & 0 \\
\hline Respiratory & 2 & 0 \\
\hline Pleural effusion & 1 & 1 \\
\hline Ocular & 0 & 1 \\
\hline Joint swelling & 0 & 1 \\
\hline$>1$ manifestation of PR & 4 & 2 \\
\hline
\end{tabular}

Of the $50 \mathrm{HIV}+\mathrm{TB}+$ patients studied the median CD4 count at the time of TB diagnosis was 119 cells/ $\mu$ l (range 2-831) and HIV viral load was $5.5 \mathrm{log}$ copies/ml (range 1.69-5.9). Nine did not have HAART during treatment for TB, 13 took HAART both before and after the diagnosis of TB, and 28 received HAART subsequent to the diagnosis of TB.

The 28 HIV+TB+ patients who received HAART only after starting antituberculosis treatment were analysed separately. The data for this group are shown in table 3. Eight of the 28 patients $(29 \%)$ had PR. Their median CD4 count at TB diagnosis was 58 cells/ $\mu$ l (range 30-143) and the viral load was $5.5 \mathrm{log}$ copies/ml (range 5.2-5.9). No association was found between PR and baseline CD4 count or CD4 response on HAART. In this group PR was significantly associated with starting HAART within 6 weeks of TB diagnosis $(p=0.03)$. PR was more common in those with disseminated disease (6/13 with dissemination $v 2 / 15$ without dissemination; $\mathrm{p}=0.09)$ and viral load suppression to $<400$ copies $/ \mathrm{ml}$ within 6 months $(p=0.3)$. However, none of these reached statistical significance.

\section{DISCUSSION}

Our results, based on a population of patients with a range of clinical disease, suggest that PR during treatment for TB is 
Table 3 Factors associated with paradoxical reaction (PR) in patients starting HAART after commencing antituberculous treatment

\begin{tabular}{|c|c|c|}
\hline & No $(\%)$ with PR & p value ${ }^{*}$ \\
\hline \multicolumn{3}{|l|}{ CD4 count at starting HAART } \\
\hline$>50$ cells $/ \mathrm{mm}^{3}$ & $4 / 17(24 \%)$ & 0.7 \\
\hline$<50$ cells $/ \mathrm{mm}^{3}$ & $4 / 11(36 \%)$ & \\
\hline \multicolumn{3}{|l|}{ Viral load at starting HAART } \\
\hline$<5.5$ log copies $/ \mathrm{ml}$ & $3 / 14(21 \%)$ & 0.7 \\
\hline$>5.5 \log$ copies $/ \mathrm{ml}$ & $5 / 14(36 \%)$ & \\
\hline \multicolumn{3}{|l|}{ Disseminated TB } \\
\hline No & $2 / 15(13 \%)$ & 0.09 \\
\hline Yes & $6 / 13(46 \%)$ & \\
\hline \multicolumn{3}{|l|}{ CD4 cell response to HAART } \\
\hline$>100$ cells $/ \mathrm{mm}^{3}$ increase & $6 / 18(33 \%)$ & 0.7 \\
\hline$<100$ cells $/ \mathrm{mm}^{3}$ increase & $2 / 10(20 \%)$ & \\
\hline \multicolumn{3}{|l|}{ Viral load (VL) response to HAART } \\
\hline Achieved VL $<400$ copies $/ \mathrm{ml}$ & $8 / 24(33 \%)$ & 0.3 \\
\hline Did not achieve $\mathrm{VL}<400$ copies $/ \mathrm{ml}$ & $0 / 4(0 \%)$ & \\
\hline \multicolumn{3}{|c|}{ Ethnicity } \\
\hline Black African & $5 / 16(31 \%)$ & 1.0 \\
\hline Other & $3 / 12(25 \%)$ & \\
\hline \multicolumn{3}{|l|}{ Time from TB treatment to HAART } \\
\hline$<6$ weeks & $7 / 13$ (54\%) & 0.03 \\
\hline 6 weeks -6 months & $1 / 11(9 \%)$ & \\
\hline$>6$ months & $0 / 4(0 \%)$ & \\
\hline
\end{tabular}

common in both HIV infected and uninfected individuals. The observed frequency of $28 \%$ in our HIV positive patients is lower than some groups have reported since the introduction of HAART, while our rate of $10 \%$ in HIV negative patients is higher than the rate of $2 \%$ reported by Narita et al in their HIV negative comparison group. ${ }^{5}$ We believe these differences may be due to patient selection. We have investigated a typical mixed inpatient and outpatient study population with a range of TB presentations. All patients received daily selfadministered treatment and, although not formally assessed in this study, adherence to treatment in our cohort is generally excellent.

The main differences between our HIV positive and HIV negative groups were with respect to ethnicity and the presence of disseminated TB. The former reflects the demographic characteristics in our practice as a whole. We observed PR in all ethnic groups with no difference in frequency according to race, although the sample size for this analysis was small. However, there have been no reports of ethnic differences in the occurrence of PR and we do not believe that ethnicity explains the increased frequency in the HIV positive group.

We and others have shown a strong association between the use of HAART and PR. ${ }^{10}$ The underlying mechanism for this is unclear. Our data suggest that PR is independent of both baseline CD4 count and HAART mediated recovery of CD4 cells as measured in peripheral blood. However, these may not accurately reflect local immune responses in, for example, the lung which are probably more relevant. ${ }^{11}$ This might explain the reported slow recovery of $M$ tuberculosis specific $\mathrm{T}$ cell responses in blood from patients starting HAART. ${ }^{12}{ }^{13}$ There is a suggestion that a rapid reduction in HIV plasma viral load may be associated with PR, as found by Navas et al. ${ }^{10}$

In practice, the association between early initiation of HAART and PR creates a dilemma for the clinician treating TB/HIV co-infection. Against this must be balanced the risk of further opportunistic infections if HAART is delayed. A large study of co-infected patients showed that four of 188 had a further AIDS defining illness (ADI) within the first 2 months of TB treatment. ${ }^{14}$ Those at particular risk of further ADI included patients with a CD4 count below 100 cells/ $\mu \mathrm{l}$ and patients not receiving HAART. Although our study could not investigate the reasons which lead physicians to decide when to prescribe HAART after TB treatment and hence we cannot rule out confounding factors, our data suggest that delaying HAART to avoid PR may be advisable in the presence of disseminated TB but not necessary in those with low CD4 counts alone. A flaw in this strategy may be the difficulty in accurately assessing the TB burden in many patients with advanced immunosuppression.

In HIV negative patients admitted for treatment of TB, restoration of skin test responses to PPD after 2 weeks of antituberculosis treatment have been reported..$^{15}$ This has been ascribed to reversal of immunosuppression due to TB itself. However, improved nutritional status and alcohol cessation, which might be most marked in those admitted for treatment, have been shown to affect cell mediated immunity ${ }^{16}$ and thus may also play an important part.

Disseminated TB-which is seen more frequently in HIV infected TB patients ${ }^{17}$ — was associated with PR, although this did not reach statistical significance. This may not only reflect the importance of immune status, as discussed above, but also suggests that overall mycobacterial load can influence the development of PR. Campbell and Dyson ${ }^{3}$ proposed that rapid killing of bacilli by effective antituberculous treatment can cause the release of large amounts of tuberculoprotein and other cell wall products. The ability of such materials to elicit a severe and potentially fatal inflammatory response was described first by Koch himself. ${ }^{18}$ It is logical to assume that the overall inflammatory response to $M$ tuberculosis reflects both the number and function of appropriate immune cells and the amount of antigen that they encounter. The severity and frequency of PR would therefore be expected to increase if disseminated or extensive single organ disease was present.

We have shown that PR is a common phenomenon during TB treatment in a combined inpatient and outpatient population, regardless of HIV antibody status. However, it is seen more frequently in co-infected patients receiving HAART. The reasons for this remain unclear, although the timing of HAART initiation in relation to TB diagnosis appears to be important, as does the presence of disseminated disease. Our study is limited by its retrospective nature and relatively small size. Results that do not achieve statistical significance at the 5\% level may become significant with a larger sample. We believe that this warrants further prospective, clinical, and laboratory investigation.

\section{Authors' affiliations}

R A M Breen, H Bettinson, S Dart, M Johnson, $M$ Lipman, Department of Thoracic and HIV Medicine, Royal Free Hospital, London, UK

B Bannister, Department of Infectious Diseases, Royal Free Hospital, London, UK

C Smith, Department of Primary Care and Population Sciences, Royal Free and University College Medical School, London, UK

Funding: None

\section{REFERENCES}

1 Chloremis CD, Padiatellis C, Zoumboulakis D, et al. Transitory exacerbation of fever and roentgenographic findings during treatment of tuberculosis in children. Am Rev Tuberc 1955;72:527-36.

2 Silver CP, Steel SJ. Mediastinal lymphatic gland tuberculosis in Asian and coloured immigrants. Lancet, $1961 ; i, 1254-6$.

3 Campbell IA, Dyson AJ. Lymph node tuberculosis: a comparison of various methods of treatment. Tubercle 1977;58:171-9.

4 Onwubalili JK, Scott GM, Smith H. Acute respiratory distress related to chemotherapy of advanced pulmonary tuberculosis: a study of two cases and review of the literature. Q J Med 1986;59:599-610.

5 Narita M, Ashkin D, Hollender ES, et al. Paradoxical worsening of tuberculosis following anti-retroviral therapy in patients with AIDS. Am J Respir Crit Care Med 1998;158:157-61. 
6 Barry SM, Lipman $\mathrm{MCl}$, Deery AR, et al. Immune reconstitution pneumonitis following Pneumocystis carinii pneumonia in HIV-infected subjects. HIV Med 2002;3:207-1 1

7 Race EM, Adelson-Mitty J, Kriegel GR, et al. Focal mycobacterial lymphadenitis following initiation of protease-inhibitor therapy in patients with adanced HIV-1 disease. Lancet 1998;351:252-5

8 Zegans ME, Walton RC, Holland GN, et al. Transient vitreous inflammatory reactions associated with combination antiretroviral therapy in patients with AIDS and cytomegalovirus retinitis. Am J Ophthalmol 1998; 125:292-300.

9 Wendel KA, Alwood KS, Gachuhi R, et al. Paradoxical worsening of tuberculosis in HIV-infected persons. Chest 2001;120:193-7.

10 Navas E, Martin-Davila P, Moreno L, et al. Paradoxical reactions of tuberculosis in patients with the acquired immunodeficiency syndrome who are treated with highly active antiretroviral therapy. Arch Intern Med 2002;162:97-9.

11 Barry SM, Lipman MCl, Bannister B, et al. Purified protein derivative-activated type 1 cytokine-producing CD4+ T lymphocytes in the lung: a characteristic feature of active pulmonary and non-pulmonary TB. J Infect Dis 2003; 187:243-50

12 Li TS, Tubiana R, Katlama C, et al. Long-lasting recovery in CD4 T-cell function and viral-load reduction after highly active antiretroviral therapy in advanced HIV-1 disease. Lancet 1998;351:1682-6.

13 Schluger NW, Perez D, Liu YM. Reconstitution of immune responses to tuberculosis in patients with HIV infection who receive antiretroviral therapy. Chest 2002; 122:597-602

14 Dean GL, Edwards SG, Ives NJ, et al. Treatment of tuberculosis in HIV-infected persons in the era of highly active antiretroviral therapy. AIDS 2002; 16:75-83.

15 Rooney JJ, Crocco JA, Kramer S, et al. Further observations of tuberculin reactions in active tuberculosis. Am J Med 1976;60:517-22.

16 Watzl B, Watson RR. Role of alcohol abuse in nutritional immunosuppression. J Nutr 1992;122:733-7.

17 Elliott AM, Luo N, Tembo G, et al. Impact of HIV on tuberculosis in Zambia: a cross sectional study. BMJ 1990;301:412-5.

18 Koch R. Weitere Mittheilungen uber ein Heilmittel gegen Tuberculose. Dtsch Med Wochenschr 1890;16:1029.

\section{Making Health Care Safer 2004}

21-22 October 2004

Royal College of Physicians, London

A two day conference for all professionals dedicated to providing safer health care for all.

Register now! Early booking discount available.

See website for details: www.quality.bmipg.com 